\title{
Finding treatment success in patients unfit for radical cystectomy
}

\author{
Michael Leveridge, MD, FRCSC \\ Department of Urology, Department of Oncology, Kingston General Hospital/Queen's University, Kingston, ON, Canada
}

Cite as: Can Urol Assoc J 2017;11 (1-2):31-2. http://dx.doi.org/10.5489/cuaj.4409

See related article on page 24 .

$\mathrm{B}$ ladder cancer is a disease of the elderly, whose incidence continues to rise along with age at rate of up to 296 per 100000 men and 74 per 100000 women aged 85 and above. ${ }^{1}$ Applying our standard treatment decisions to the elderly is fraught due to their rise in comorbidities, which obviate or dramatically increase the risks of treatment. McPherson et al from Western University have studied these highest-risk patients and found that trimodal therapy that avoids cystectomy can provide very reasonable outcomes with modest toxicity. ${ }^{2}$

Urologists are typically the first point of contact in diagnosing muscle-invasive bladder cancer (MIBC) and act as de facto quarterbacks for subsequent referral and care. Our weapon of choice has always been cystectomy; it is the standard for curative management, controls local disease, and decreases the complexity of followup. The authors point out though that morbidity and mortality rise from the already significant baseline in octogenarians. More recent population data from Ontario, in fact, show that 30- and 90-day mortality after radical cystectomy in octogenarians is $6 \%$ and $15 \%$, respectively. ${ }^{3}$ Three-year overall and diseasefree survival in this age group was about $35 \%$ and $40 \%$, respectively (after cystectomy). The current study cohort, explicitly in worse medical condition than selected-forcystectomy patients nonetheless fared better. It is heartening to see therapeutic options that seem to carry less early risk to the patient.

This study is also notable for the contortions undertaken to ensure that patients received chemotherapy in some form, even if the standard cisplatin was contraindicated. A weakness here is that we don't know the denominator of all elderly patients at this centre (from those fit for surgery through those whose comorbidities leapfrog the study cohort, too ill for chemoradiation), but it is clear that an effort to treat medically tenuous patients (25\% Eastern Cooperative Oncology
Group [ECOG] 2+, only $12.5 \%$ ECOG 0) was made. Prior studies have shown decreased medical oncology referral and use of chemotherapy in older patients. ${ }^{3-5}$ Dash et al showed that over $40 \%$ of bladder cancer patients over age 70 were cisplatin-ineligible, while Gupta et al showed lower use of cisplatin in chemotherapy-treated MIBC patients in a Surveillance, Epidemiology, and End Results (SEER)Medicare analysis (53\% for patients $<75$ years vs. $30 \%$ for those $80+$ years) ${ }^{6,7}$ The presence of hydronephrosis in onethird of McPherson et al's cohort suggests a similar level of risk.

Numbers are too low to confirm that a conventional treatment course of radiotherapy should be applied over a lower-dose protocol, but the toxicity data and trends here suggest that an effort to maximize local therapy may benefit survival without Grade 3-4 toxicity. In a phase 3 study of radiotherapy vs. chemoradiotherapy (as the authors here discuss), a $62 \mathrm{~Gy} / 32$ fraction protocol was not significantly different from a $55 \mathrm{~Gy} / 20$ fraction course (hazard ratio favouring chemotherapy 0.63 [95\% confidence interval $0.41-0.98]$ vs. $0.77[0.43-1.36])^{8}$

Finally, while these encouraging results are seen in the setting of a multidisciplinary, tertiary cancer centre (and in comparison to population data lend support to such centralization of complex care), it is notable that only 31 patients $(78 \%)$ appear to have had a surveillance cystoscopy and followup imaging was left to the "discretion of the clinical team. ${ }^{2}$ Rigorous adherence to a followup schedule may eke out a small additional benefit to the outcomes noted.

We are limited somewhat by the unknown selection criteria and small numbers, but this study does reveal that acceptable survival and local control outcomes, historically comparable to radical cystectomy, can be achieved in unwell, older patients. Patients with impaired performance status or some renal dysfunction clearly should not be discounted from receiving chemotherapy. Importantly, these data do not suggest that cystectomy is unwise or unacceptably risky in healthy octogenarians, but it is a powerful tool that comes at a cost that may be untenable in the old or infirm. Knowing that safe and effective treatment may still 
be afforded these patients, however, may make urologists feel less compelled to offer high-risk surgery to the patients at highest risk of harm.

Competing interests: The author reports no competing personal or financial interests.

\section{References}

1. Shariat SF, Milowsky M, Droller MJ. Bladder cancer in the elderly. Urol Oncol 2009;27:653-67. https://doi.org/10.1016/.j.urolonc.2009.07.020

2. McPherson VA, Rodrigues $G$, Bauman $G$, et al. Chemoradiotherapy in octogenarians as primary treatment for muscle-invasive bladder cancer. Can Urol Assoc J 2017;11:24-30. http://dx.doi.org/10.5489/ cuaj.4008

3. Leveridge MJ, Siemens DR, Mackillop WJ, et al. Radical cystectomy and adjuvant chemotherapy for bladder cancer in the elderly: A population-based study. Urology 2015; 85:791-8. https://doi.org/10.1016/i. urology.2014.12.027

4. Booth CM, Siemens DR, Pei Y, et al. Patterns of referral for perioperative chemotherapy among patients with muscle-nnvasive bladder cancer: A population-based study. Urol Oncol 2014;32:1200-8. https://doi.org/10.1016/i.urolonc.2014.05.012

5. Nielsen ME, Shariat SF, Karakiewicz PI, et al. Advanced age is associated with poorer bladder cancer-specific survival in patients treated with radical cystectomy. Eur Urol 2007;51:699-708. https://doi.org/10.1016/i.eururo.2006.11.004

6. Dash A, Galsky MD, Vickers AJ, et al. Impact of renal impairment on eligibility for adjuvant cisplatinbased chemotherapy in patients with urothelial carcinoma of the bladder. Cancer 2006;107:506-13. https://doi.org/10.1002/cncr.22031

7. Gupta A, Long JB, Chen J, et al. Risk of vascular toxicity with platinum-based chemotherapy in elderly patients with bladder cancer. J Urol 2016;195:33-40. https://doi.org/10.1016/i.juro.2015.08.088

8. James ND, Hussain SA, Hall E, et al. Radiotherapy with or without chemotherapy in muscle-invasive bladder cancer. N Engl J Med 2012;366:1477-88. https://doi.org/10.1056/NEJMoal 106106

Correspondence: Dr. Michael Leveridge, Department of Urology, Department of Oncology, Kingston General Hospital/Queen's University, Kingston, 0N, Canada; leveridm@kgh.kari.net; twitter: @_TheUrologist_

\section{${ }^{\circ} \mathrm{XGEVA}$ \\ (denosumab)}

Indication and clinical use:

- XGEVA ${ }^{\circledR}$ is indicated for reducing the risk of developing skeletal-related events (SREs) in patients with bone metastases from breast cancer, prostate cancer, non-small cell lung cancer, and other solid tumours.

- Not indicated for reducing the risk of developing skeletal-related events in patients with multiple myeloma.

- Not indicated for reducing the risk of developing skeletal-related events in pediatric patients.

\section{Contraindications:}

- In patients with pre-existing hypocalcemia, which must be corrected prior to initiation.

\section{Most serious warnings and precautions:}

Osteonecrosis of the jaw (ONJ): In clinical trials, the incidence of ONJ was higher with longer duration of exposure. In patients with risk factors for $0 \mathrm{NJ}$, an individual risk/benefit assessment should be performed before initiating therapy with XGEVA. An oral exam should be performed and a dental exam with appropriate preventive dentistry is recommended prior to treatment with XGEVA, especially in patients with risk factors for ONJ. Avoid invasive dental procedures while receiving XGEVA. In patients who develop ONJ during treatment with XGEVA, a temporary interruption of treatment should be considered based on individual risk/benefit assessment until the condition resolves.

\section{Other relevant warnings and precautions:}

- Do not use concurrently with Prolia

- Do not use concurrently with bisphosphonates

- Hypocalcemia has been reported (including severe symptomatic hypocalcemia and fatal cases). Monitor calcium prior to the initial dose, within two weeks after the initial dose, and if suspected symptoms of hypocalcemia occur. Administer adequate calcium, vitamin $D$, and magnesium, as necessary. If hypocalcemia occurs while receiving XGEVA, additional short-term calcium supplementation and additional monitoring may be necessary.

- Caution on risk of hypocalcemia and accompanying increases in parathyroid hormone in patients with renal impairment

- Skin infections

- Hypersensitivity reactions including anaphylaxis

- Atypical femoral fractures

- Not recommended for use in pregnant women. Women should not become pregnant during treatment and for at least 5 months after the last dose of XGEVA.

\section{For more information:}

Please consult the Product Monograph at

http://www.amgen.ca/Xgeva_PM.pdf for important information relating to adverse reactions, drug interactions, and dosing that have not been discussed here.

The Product Monograph is also available by calling Amgen Medical Information at 1-866-502-6436.

\section{Fizazi et al. study ${ }^{2}$}

Phase 3, randomized, double-blind, double-dummy, active-controlled study. Patients with castrate-resistant prostate cancer and bone metastases $(n=1901)$ received either $120 \mathrm{mg}$ XGEVA SC Q4W (once every 4 weeks) ( $n=950)$ or $4 \mathrm{mg}$ zoledronic acid IV Q4W ( $n=951)$. The primary outcome measure was to demonstrate non-inferiority of time to first on-study SRE as compared to zoledronic acid. The secondary outcome measures were superiority of time to first on-study SRE and superiority of time to first and subsequent SREs. An SRE is defined as any of the following: pathologic fracture, radiation therapy to bone, surgery to bone or spinal cord compression.

References:

1. XGEVA ${ }^{\circledast}$ Product Monograph, Amgen Canada, 2015.

2. Fizazi $\mathrm{K}$, et al. Denosumab versus zoledronic acid for treatment of bone metastases in men with castration-resistant prostate cancer: a randomized, double-blind study. Lancet. 2011;377(9768):813-822.
AMCEN

Oncology
๑) 2016 Amgen Canada Inc. All rights reserved.

ק. MEMBER OF INNOVATIVE MEDICINES CANADA 